Arch Plast Surg 2013;40:165-7.

5. Idriss MH, Kazlouskaya V, Malhotra S, et al.

Phosphohistone-H3 and Ki-67 immunostaining in

cutaneous pilar leiomyoma and leiomyosarcoma

(atypical intradermal smooth muscle neoplasm).J

Cutan Pathol 2013;40:557-63.

\section{The Rare Presentation of Extraskeletal Ewing's Sarcoma on the Forehead}

Sung Hoon $\mathrm{Choi}^{1}$, Young Joon $\mathrm{Kim}^{1}$, Hoon $\mathrm{Kim}^{1}$, Hyun-Jung Kim², Sang Hyun Nam ${ }^{1}$,

Young Woong Choi ${ }^{1}$

Departments of ${ }^{1}$ Plastic and Reconstructive Surgery and ${ }^{2}$ Pathology, Sanggye Paik Hospital, Inje University College of Medicine, Seoul, Korea

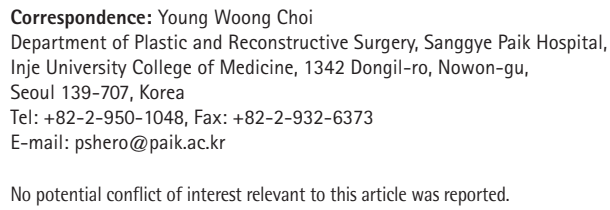

No potential conflict of interest relevant to this article was reported.

Received: 6 Mar 2014 • Revised: 24 Mar 2014• Accepted: 27 Mar 2014 pISSN: 2234-6163・ elSSN: 2234-6171

http://dx.doi.org/10.5999/aps.2015.42.1.100 • Arch Plast Surg 2015;42:100-102

Copyright (C) 2015 The Korean Society of Plastic and Reconstructive Surgeons

This is an Open Access article distributed under the terms of the Creative Commons

Attribution Non-Commercial License (http://creativecommons.org/licenses/by-nc/3.0/) which permits unrestricted non-commercial use, distribution, and reproduction in any medium, provided the original work is properly cited.

Fig. 1.

Preoperative photograph showing a non-tender mobile soft tissue (black arrow) mass on the forehead.
Extraskeletal Ewing's sarcoma (EES) is a rare round cell malignant neoplasm with rapid growth and an uncharacterized mesenchymal cell origin, and it is histologically similar to Ewing's sarcoma (ES) arising from bone [1]. EES was first described as a paravertebral 'round cell' tumor morphologically resembling ES of bone by in 1969 [1,2]. In 1975, a review of 39 patients was reported about malignant soft tissue tumors not arising from bone but with histomorphologic characteristics similar to primary osseous ES [1,2].

The sites of involvement varied, yet there appeared to be a tendency to involve the paravertebral region, intercostal area, lower extremities and pelvis [1]. EES rarely occurs in the head and neck region, with only five cases reported in a series of 118 patients in the four largest series in the English literature $[1,3]$. Furthermore, another report indicated that only 5\% to $11 \%$ of EES cases occur in the head and neck region, and the nose, eyelid, nasopharynx, parotid gland, scalp, and parapharyngeal space have been described $[3,4]$.

However, we encountered EES on the forehead. To the best of our knowledge, there have been no reports regarding EES originating from the forehead. Two years ago, a 35-year-old male patient presented with a palpable and non-tender mass. The mass size was $2 \times 2 \mathrm{~cm}$, and there were no other problems. Excision was performed in a local clinic one year ago. After excision, he had no clinical symptoms. However, 3 months ago, a $1 \times 1 \mathrm{~cm}$ mobile, protruding mass recurred on the patient's forehead (Figs. 1, 2). The patient visited our department for further evaluation and treatment. At the initial physical examination, lymph node enlargement in the neck, weight loss, tenderness and other clinical problems were not observed.

Radiologic evaluation demonstrated no invasion or any destruction of the frontal bone. X-ray examination showed a soft tissue mass with muscle tissue invasion that was without calcification or onion skin-like radiation characteristics, which are specific to osseous ES (Fig. 3).

Under local anesthesia, excisional biopsy was performed. An approximately $1.0 \times 1.0 \mathrm{~cm}$ tissue mass was excised. The pathologic report revealed monomorphic proliferation of small, blue, round cell tumors with scanty cytoplasm and the absence of rosette-like structures (Fig. 4). Immunohistochemical staining demonstrated that the tumor cells were positive for vimentin, CD99, CD56, and neuron- 

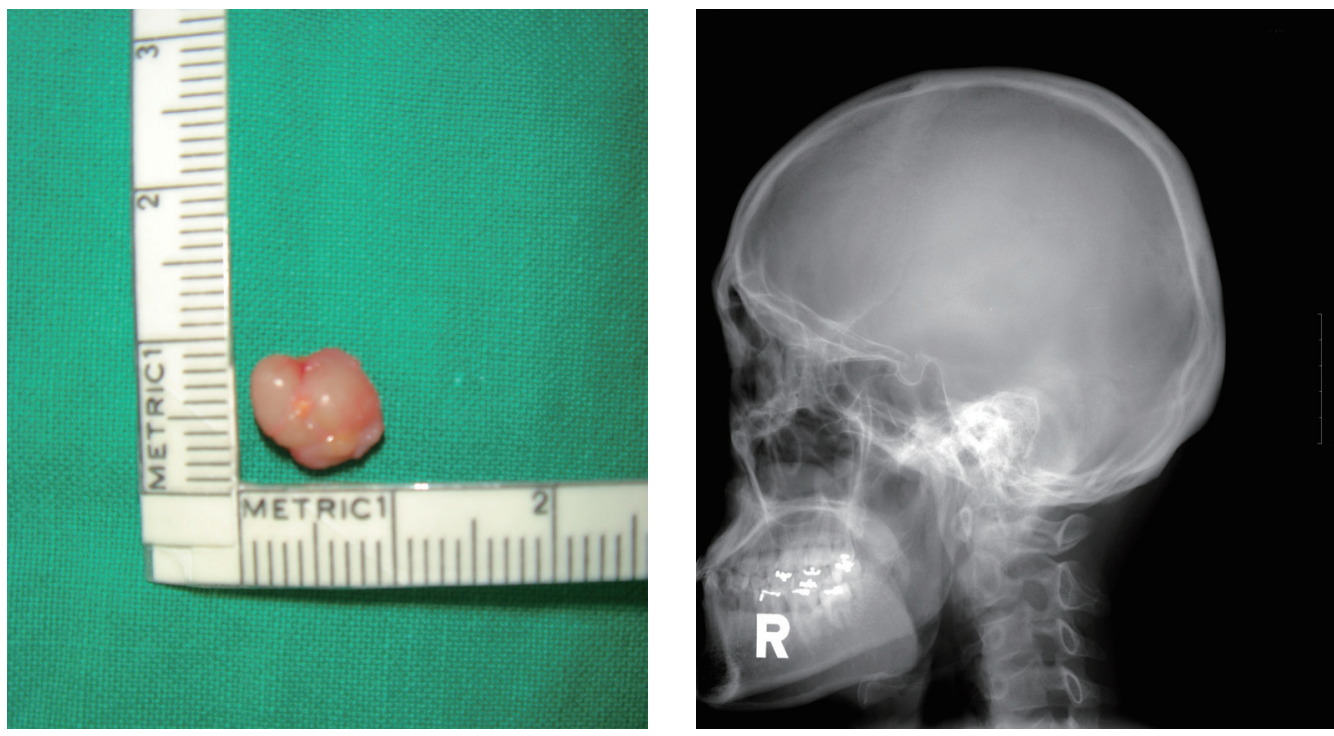

Fig. 2.

Photograph of the grayish brown excised mass, sized

$1 \times 1 \mathrm{~cm}$.

specific enolase (NSE) (Fig. 5) but negative for chromogranin, synaptophysin, myeloperoxidase, and common leukocyte antigen. Periodic acid-Schiff (PAS) staining was negative.

The tumor was finally diagnosed as EES by a pathologist. After surgical excision, the patient was required to follow-up for further evaluation. We therefore recommended additional studies, including facial computed tomography, bone scan, and magnetic resonance imaging, among others. We also recommended adjuvant chemo-radiotherapy in the oncology department to ensure the best outcome. However, the patient refused all studies and treatment. We tried to contact him with an effort, but lost contact to him because his phone number was changed. And he did not appear to our department. EES has non-specific characteristics regarding its symptoms and signs as well as a low incidence rate, and it typically has an aggressive course with a high incidence of recurrence. However, it has a high tendency to spread locally, infiltrating surrounding fascial planes and invading muscle and bone [3]. Distant metastasis is common, and most frequently occurs in the lung [4]. Therefore, an early definitive diagnosis is important.

The rarity of EES and locations at uncommon sites may lead to a delay in clinical diagnosis and appropriate treatment and management. Therefore, when clinicians observe a soft tissue mass that grows rapidly within a few months, EES should be clinically suspected, with a comprehensive work-up such as radiologic and pathologic examinations necessary to confirm the diagnosis.

Before the development of appropriate medical treatment, the prognosis of EES was poor. In a study, the 1-year survival rate was only $40 \%$ [1]. Therefore, various therapeutic treatment plans have been developed. In a recent study, the two-year survival rate was $65 \%$, although $88 \%$ of the patients were treated with chemo-radiotherapy [1]. Furthermore, another report described an $82 \%$ 2-year survival rate [1]. If radical excision with adjuvant chemoradiotherapy is received, the survival rate of EES can

Although clinical and radiographic evaluations are important in the early diagnosis of EES, the definitive identification is made in conjunction with pathologic findings [2]. Therefore, the diagnosis of EES is typically made after excision. In our case, the patient refused all recommendations. Therefore, patients must be informed about further evaluation and further surgery or chemo-radiotherapy to ensure better compliance if the mass has a possibility of being EES.

In our case, the pathologic report revealed monomorphic proliferation of small round cell tumors and the absence of rosette-like structures. The differential diagnosis includes small round cell tumors such as rhabdomyosarcoma, neuroblastoma, primitive peripheral neuroectodermal tumor (PNET), Merkel cell carcinoma and lymphoma $[1,3,5]$. Antibody staining for CD99 helps to confirm the diagnosis. be increased dramatically.
Fig. 3.

Radiologic evaluation of a skull lateral X-ray showing no abnormal calcification or bony erosion. 


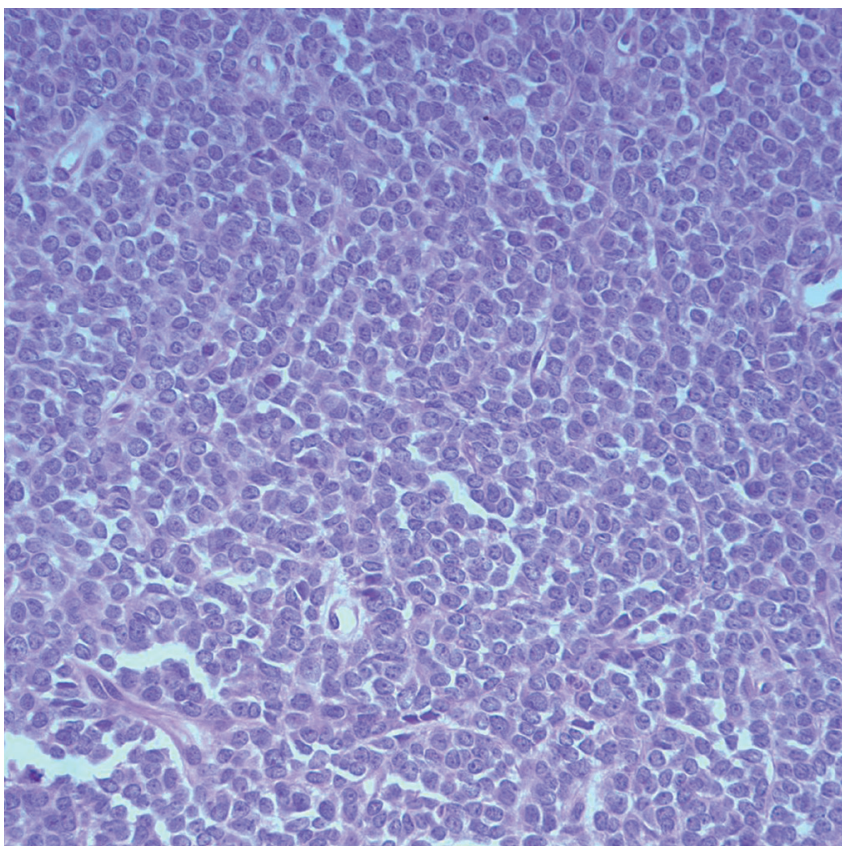

Fig. 4.

Histopathology showing monomorphic small, round, blue cells with scant cytoplasm and prominent nucleoli $\left(H \& E_{1} \times 400\right)$.

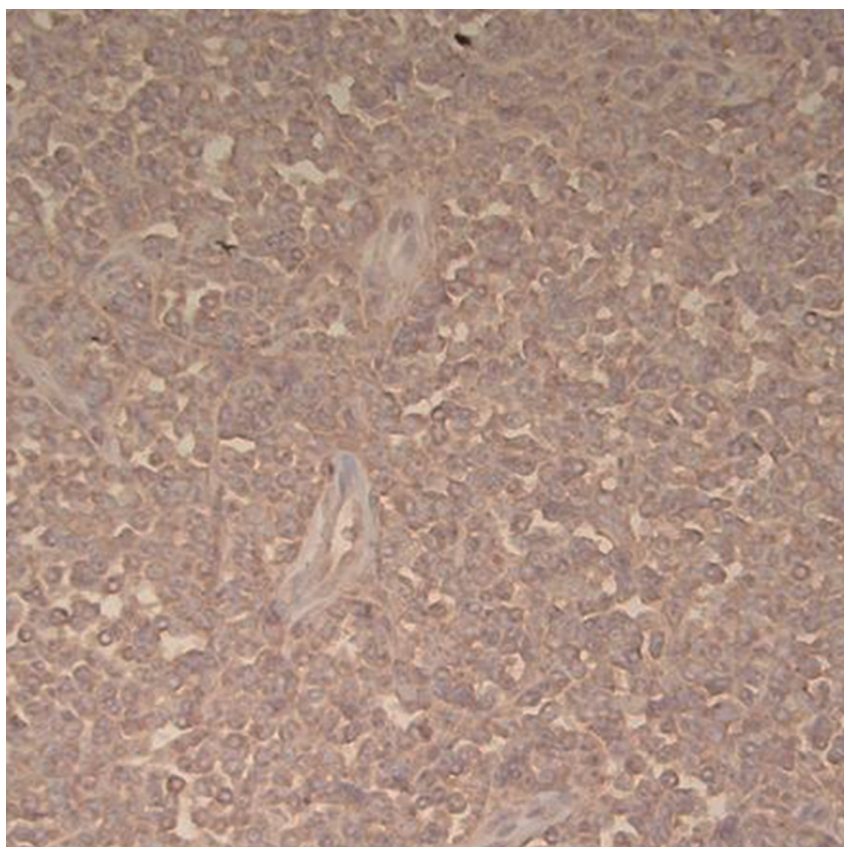

Fig. 5.

Immunohistochemical staining revealed weak but diffuse positivity for CD99 ( × 400).
PNET shows neuroectodermal differentiation, with rosette formation and positive immunohistochemical staining in the nucleus for NSE, neurofilament and synaptophysin. In our case, even NSE was positive following immunohistochemical staining, but there was no evidence of neuroectodermal differentiation. Moreover, PAS is typically positive in EES, but PAS was negative in our case [5]. Despite the histomorphologic difficulties in differentiating this tumor from other soft tissue malignancies, EES must be diagnosed earlier to allow initiation of a treatment plan to increase the survival rate.

In conclusion, EES is a rare soft tissue sarcoma and has an aggressive course with a high incidence of recurrence. However, EES/PNET is sensitive to multimodal treatment. Early awareness and treatment of this rare disease and wide resection, followed by chemo-radiotherapy, might improve long-term patient survival. In our case, we could not treat the patient to this end. Therefore, before the operation, patients should be informed about further treatment and management for EES. Additionally, special consideration must be given to rapidly growing soft tissue masses that present with or without tenderness.

\section{References}

1. Chao TK, Chang YL, Sheen TS. Extraskeletal Ewing's sarcoma of the scalp. J Laryngol Otol 2000;114:73-5.

2. Kennedy JG, Eustace S, Caulfield R, et al. Extraskeletal Ewing's sarcoma: a case report and review of the literature. Spine (Phila Pa 1976) 2000;25:1996-9.

3. Agir H, Brasch HD, Tan ST. Extra-skeletal Ewing's sarcoma of the submandibular gland. J Plast Reconstr Aesthet Surg 2007;60:1345-8.

4. Ng SH, Ko SF, Cheung YC, et al. Extraskeletal Ewing's sarcoma of the parapharyngeal space. Br J Radiol 2004; 77:1046-9.

5. Lee WJ, Kim MS, Kang SM, et al. Primary cutaneous extraskeletal Ewing's sarcoma. Korean J Dermatol 2008; 46:1427-31. 